reperfusão.A RCR diminuiu após 120 minutos de isquemia retornando a níveis semelhantes ao controle após a reperfusão. Conclusão: A CHE foi sensível para indicar a lesão isquêmica, sugerindo irreversibilidade da lesão. Já a RCR foi mais sensível no sentido de detectar a reversibilidade da lesão isquêmica após a reperfusão.

DESCRITORES: Colinesterase. Função mitocondrial.Transplante de fígado.Isquemia. Reperfusão.

Correspondence to:

Orlando Castro-e-Silva Jr Conflito de interesse: nenhum.

Fonte de financiamento:FAPESP

Rua Campos Salles 890, 9 andar Cep: 14015-110

Centro, Ribeirão Preto, São Paulo, Brazil.

$55166022871 / 55166100626$

Email: orlandocsj@hotmail.com

15 - ARTIGORETROSPECTIVO

\title{
Adrenalectomia laparoscópica: análise de 11 pacientes $^{1}$
}

Ricardo Brianezi Tiraboschi², André Luis Alonso Domingos², Rodolfo Borges Reis ${ }^{3}$, Tiago Borelli Bovo ${ }^{2}$ Haylton Jorge Suaid ${ }^{3}$, Adauto José Cologna ${ }^{3}$, Antonio Carlos Pereira Martins ${ }^{3}$

Tiraboschi RB, Domingos ALA, Reis RB, Bovo TB, Suaid HJ, Cologna AJ, Martins ACP. Adrenalectomia laparoscópica: análise de 11 pacientes. Acta Cir Bras [serial online] 2003 vol 18 suppl 5. Disponível em www.scielo.br/acb

RESUMO - Objetivo: Analisar a experiência inicial da Divisão de Urologia do HCFMRP-USP na adrenalectomia transperitoneal videolaparoscópica. Métodos: Análise retrospectiva de 11 casos de adrenalectomia transperitoneal laparoscópica realizados de fevereiro de 1999 a março de 2003 sendo 3 em homens( 27\%) e 8 em mulheres (73\%), idade média de 40,2 $\pm 13,1$ anos. Os pacientes apresentavam os diagnósticos seguintes: adenoma -5 , síndrome de Cushing -3 , feocromocitoma - 1, hiperaldestorismo - 1 e síndrome de Carney - 1. Resultados: A cirurgia foi bilateral em 05 pacientes $(45,4 \%)$ e unilateral em 06 pacientes $(54,6 \%)$, destes 04 à direita $(36,4 \%)$ e 02 à esquerda $(18,2 \%)$. O tempo médio de internação foi de $3,6 \pm 1,1$ dias, o tempo médio de cirurgia foi de $220,5 \pm 103,7$ minutos e a taxa de conversão foi de $18,2 \%$. Conclusão: Os resultados apresentados são similares aos relatados pela literatura, demonstrando que a adrenalectomia videolaparoscópia pode ser realizada de maneira segura e eficiente com benefícios: tempo cirúrgico aceitável, rápida recuperação pós-operatória e alta precoce.

DESCRITORES: Adrenalectomia, laparoscopia. Adenoma da adrenal. Cushing. Conn. Carey.

\section{INTRODUÇÃO}

O uso de técnicas minimamente invasivas tem mudado o acesso cirúrgico à glândula adrenal. Desde sua primeira descrição, em 1991 por Gaygner ${ }^{1}$, a adrenalectomia videolaparoscópica tem se difundido com ampla aceitação mundial ${ }^{2}$. Antes do advento da laparoscopia o acesso cirúrgico tradicional geralmente se fazia por ampla incisão na pele para exposição adequada de um órgão pequeno e friável com abundante e delicada vascularização, situada no retroperitô$n^{n}{ }^{3}$. Na última década vários trabalhos foram publicados a respeito dos benefícios da adrenalectomia videolaparoscópica ${ }^{2,4,5}$. Atualmente é considerada o tratamento padrão para a maioria dos pacientes com patologias cirúrgicas da adrenal, incluindo tumores e o feocromocitoma ${ }^{4,5}$. O procedimento está associado com menor período de convalescência, menor dor pós-operatória, internação hospitalar reduzida e retorno precoce à atividade funcional do paciente ${ }^{6}$. O objetivo deste trabalho é mostrar a experiência inicial da disciplina de Urologia do HCFMRP-USP.

\section{MÉTODOS}

Análise retrospectiva dos casos de adrenalectomia laparoscópica realizada de fevereiro de 1999 a março de 2003 no HCFMRP-USP. Os procedimentos foram realizados via transperitoneal. Os pacientes foram posicionados em decúbito lateral. O pneumoperitôneo foi obtido com punção abdominal com agulha de Veress e insuflado dióxido de carbono (CO2) na pressão de 12-14 mmHg. $\mathrm{O}$ acesso à cavidade peritoneal foi obtido inicialmente através de uma pequena incisão transversa situada na linha medioclavicular na altura da cicatriz umbilical, onde foi inserido trocarte de $10 \mathrm{~mm}$ e colocado a câmera. Três portes adicionais de 5 ou $10 \mathrm{~mm}$ foram então colocados: na linha médioclavicular aproximadamente dois dedos abaixo da margem costal, na linha axilar anterior na altura da cicatriz umbilical e linha axilar posterior.

$\mathrm{Na}$ dissecção laparoscópica, numerosas pequenas artérias e veias são encontradas e devem ser controladas tanto por eletrocauterização ou clipagem pois as adrenais têm suprimento sanguíneo extremamente rico com ramos arteriais da aorta, artéria frênica inferior e artéria renal. A veia adrenal origina-se na medula e desemboca na veia cava no lado direito e na veia renal do lado esquerdo ${ }^{3}$

$\mathrm{Na}$ adrenalectomia direita o peritôneo é incisado e rebatido inferiormente, o fígado afastado para exposição adequada. A dissecção da glândula adre- nal direita começa inferior e lateral onde o tecido fibroadiposo contém pequenos vasos que são controlados. A veia adrenal entrando na veia cava é identificada e dissecada precocemente, é então duplamente ligada com clipes metálicos e seccionada. A dissecção prossegue superiormente no tecido adiposo entre a glândula adrenal e a face lateral da veia cava inferior, os vasos encontrados são cuidadosamente ligados. A musculatura póstero-inferior do diafragma pode ser vista neste ponto. A dissecção continua inferiormente até a face ínfero-medial da glândula quando é liberada da fáscia de Gerota. A glândula adrenal é removida num saco endoscópico através de um dos portes. É realizada então a revisão da hemostasia.

Para o acesso à adrenal esquerda, a flexura esplênica deve ser incisada e o cólon rebatido inferiormente, geralmente o baço não necessita de afastamento. A dissecção do tecido ao redor da face posterior da cauda do pâncreas ajuda a definir a borda anterior da adrenal esquerda. A dissecção continua póstero-inferiormente no tecido fibroadiposo entre a adrenal e o rim e prossegue anteriormente com atenção para a localização da veia adrenal esquerda.

Os diagnósticos estão apresentados na Tabela 1 . Nenhum dos tumores apresentava diâmetro superior a $4 \mathrm{~cm}$.

1. Pesquisa realizada no Hospital das Clinicas - FMRP-USP

2. Medico Residente de Urologia / Hospital das Clinicas - FMRP-USP

3. Professores e Assistentes de Urologia Hospital das Clinicas - FMRP-USP 
Tabela 1: Diagnósticos dos pacientes.

\begin{tabular}{l|c|c}
\hline \multicolumn{1}{c|}{ DIAGNóSTICO } & F & F\% \\
\hline Adenoma & 5 & 45,4 \\
\hline Feocromocitoma & 1 & 9,0 \\
\hline Hiperaldosteronismo & 1 & 9,0 \\
\hline Síndrome de Carney & 1 & 9,0 \\
\hline Síndrome de Cushing & 3 & 27,6 \\
\hline
\end{tabular}

A taxa de conversão foi de $18,2 \%$, tendo ocorrido em 2 pacientes: a primeira em uma paciente com diagnóstico de Síndrome de Carney submetida à adrenalectomia bilateral complicada por lesão da cauda do pâncreas durante a adrenalectomia esquerda e posterior conversão, a segunda complicação em uma paciente com diagnóstico de adenoma de adrenal submetida à adrenalectomia direita complicada por laceração da veia adrenal na inserção da veia cava com necessidade de conversão imediata. Ambas tiveram sangramento significativo, recuperação mais lenta e aumento do tempo de hospitalização. Não houve nenhum caso de morte.

Não houve necessidade de transfusão sangüínea nas cirurgias videolaparoscópicas, exceto nos 2 casos que necessitaram de conversão para cirurgia aberta.

\section{DISCUSSÃO}

O tratamento endoscópico das doenças cirúrgicas da adrenal foi muito difundido na última década sua indicação está bem estabelecida para os tumores benignos, síndrome de Cushing, feocromocitoma, hiperaldosteronismo primário ${ }^{7,8,9}$. A via laparoscópica foi proposta também para casos de câncer e em doenças metastáticas para a adrenal mas esta conduta não está estabelecida e ultimamente tem sido contra-indicada em razão do risco de carcinomatose peritoneal ${ }^{10,11,12,13}$. O procedimento tem a melhor indicação em tumores menores que $4 \mathrm{~cm}$ pois acima disto além da dificuldade técnica há o risco da presença de tumor maligno ${ }^{2,6}$.

A adrenalectomia é considerada um procedimento de técnica laparoscópica avançada. O uso de ótica angulada ou de $0^{\circ}$ e múltiplos instrumentos simultaneamente pode ser desafiador, exigindo importante curva de aprendizado ${ }^{14,15}$. Apesar disso, nossa experiência inicial de 11 casos mostrou resultados semelhantes aos da literatura: taxa de complicações e conversão, tempo cirúrgico, tempo de internação ${ }^{16,17}$, permitindo um regresso precoce do paciente ao lar e retorno a sua atividade funcional ${ }^{18}$.

\section{RESULTADOS}

Foram realizados 11 procedimentos, $03 \mathrm{em}$ homens $(27 \%)$ e 08 em mulheres $(73 \%)$. A cirurgia foi bilateral em 05 pacientes $(45,4 \%)$ e unilateral em 06 pacientes $(54,6 \%)$, tendo sido 04 à direita $(36,4 \%)$ e 02 à esquerda $(18,2 \%)$.

A idade, o tempo de internação e o tempo cirúrgico médios estão demonstrados na Tabela 2.

TABELA 2: Idade, tempo de internação e duração do ato cirúrgico.

\begin{tabular}{ll}
\hline \multicolumn{1}{c}{ PARÂMETRO } & \multicolumn{1}{c}{ TEMPO } \\
\hline Idade (anos) & $40,2 \pm 13,1(25-63)$ \\
\hline Tempo de Internação (dias) & $3,6 \pm 1,1(3-6)$ \\
\hline Tempo cirúrgico (minutos) & $220,5 \pm 103,7(90-405)$ \\
\hline
\end{tabular}

\section{CONCLUSÃO}

Nossa experiência confirma que a adrenalectomia videolaparoscópica é um método seguro e eficaz oferecendo os benefícios da cirurgia minimamente invasiva. Estes benefícios incluem tempo cirúrgico aceitável, rápida recuperação pós-operatória e alta precoce.

\section{REFERÊNCIAS}

1. Gagner M, Lacroix A, Bolte E. Laparoscopic adrenalectomy in Cushing's syndrome and pheochromocytoma. New Engl J Med 1992; 327: 1033 .

2. Gill IS. The case for laparoscopic adrenalectomy. J Urol 2001; 166:429-436.

3. Bravo EL, Steward BH. The adrenal: anatomy and physiology. Urology Update Series, 1978, lesson 2, vol.1, p.1.

4. Gill IS, Novick AC. Laparoscopic versus open adrenal surgery. AUA Update Series, 1999, lesson 33, vol. 28, p. 258.

5. Col V, de Canniere L, Collard E, Michel L, Donkier J. Laparoscopic adrenalectomy for pheochromocytoma: endocrinological and surgical aspects of a new therapeutic approach. Clin Endocrinol 1999; 50: 121.

6. Jacobs JK, Goldstein RE, Gerr RJ. Laparoscopic adrenalectomy: a new standard of care. Ann Surg 1997; 225: 495-502

7. Shen WT, Lim RC, Siperstein AE, Clark OH, Schecter WP, Hunt TK, Horn JK, Duh QY. Laparoscopic vs open adrenalectomy for the treatment of primary hyperaldosteronism. Arch Surg 1999; 134:628-31.

8. Duncan JL 3rd, Fuhrman GM, Bolton JS, Bowen JD, Richardson WS. Laparoscopic adrenalectomy is superior to an open approach to treat primary hyperaldosteronism. American Surgeon 2000; 66:932-5.

9. Kebebew E., Siperstein A.E. and Duh Q.Y., Laparoscopic adrenalectomy: the optimal surgical approach. Journal of
Laparoendoscopic \& Advanced Surgical 2001; 11: 409-13.

10. Hobart MG, Gill IS, Schweizer D, Sung GT, Bravo EL. Laparoscopic adrenalectomy for large-volume ( $>$ or $=5 \mathrm{~cm}$ ) adrenal masses. J Endourol 2000; 14: 149-54.

11. Suzuki K, Ushiyama T, Mugya S, Kageyama S, Saisu K, Fujita K. Hazards of laparoscopic adrenalectomy in patients with adrenal malignancy. J Urol 1997; 158: 2227-31.

12. Elashry OM, Clayman RV, Soble JJ, McDougall EM. Laparoscopic adrenalectomy for solitary for metachronous contralateral adrenal metastasis from renal cell carcinoma. J Urol 1997; 157: 1217-22.

13. Heniford BT, Arca MJ, Walsh RM, Gill IS. Laparoscopic adrenalectomy for cancer. Semin Surg Oncol 1999; 16: 293-306.

14. Norman J, Baker KD. Laparoscopic adrenal resection-Changing techniques for changing indications. Cancer Control 1999; 6: 96-101.

15. Higashihara E, Baba S, Nakagawa K, Murai M, Go H, Takeda M, Takahashi K, Susuki K, Fujita K, Ono Y, Ohshima S, Matsuda T, Terachi $\mathrm{T}$, Yoshida O. Learning curve and conversion to open surgery in cases of laparoscopic adrenalectomy and nephrectomy. J Urol 1998; 159:650-3.

16. Vallancien $\mathrm{G}$, Cathelineau X, Baumert H, Doublet JD, Guillonneau B. Complications of Transperitoneal Laparoscopic Surgery in Urology: Review of 1311 Procedures at a Single Center. J Urol 2002; 168:23-6.

17. Suzuki K, Ushiyama T, Ihara H, Kageyama S, Mugya S, Fugita K. Complications of laparoscopic adrenalectomy in 75 patients treated by same surgeon. Eur Urol 1999; 36: $40-7$

18. Stanford A, Upperman JS, Nguyen N, Barksdale E, Wiener ES. Surgical management of open versus laparoscopic adrenalectomy: outcome analysis. J Pediatr Surg 2002; 37:1027-9.

Tiraboschi RB, Domingos ALA, Reis RB, Bovo TB, Suaid HJ, Cologna AJ, Martins ACP. Laparoscopic adrenalectomy - analysis of 11 patients. Acta Cir Bras [serial online] 2003 vol 18 suppl 5. Available in www.scielo.br/acb

ABSTRACT - Objective: To analyze the initial experience of the Division of Urology from HCFMRP-USP on the transperitoneal laparoscopic adrenalectomy. Methods: We analyze retrospectively 11 cases of laparoscopic adrenalectomy carried out from February of 1,999 to March of 2,003. The sample included all patients operated on through this method in such period and was composed of $3(27 \%)$ men and $8(73 \%)$ women with a mean age of $40,2 \pm 13,1$ years. The patients had the following diagnosis: adenoma -5 , Cushing's syndrome -3 , feocromocytoma - 1, Conn's syndrome -1 and Carey's syndrome - 1. Results: The adrenalectomy was bilateral in $5(45.4 \%)$ patients and unilateral in $6(54.6 \%)$ being $4(36.4 \%)$ on the right side and $2(18.2 \%)$ on the left side. The mean hospital stay was 3,6 $\pm 1,1$ days and the mean operating time was $220,5 \pm 103,7$ minutes. Conversion to open surgery was required in 2 patients (18.2\%). Conclusion: The results of laparoscopic adrenalectomy were similar to those reported elsewhere and corroborate the safety and efficacy of the method resulting in an acceptable surgical time, faster postoperative recovery and shorter hospital stay. KEY WORDS: Laparoscopy. Adrenalectomy. Adenoma. Cushing. Conn. Carey.

Endereço para correspondência:

Antonio Carlos Pereira Martins

Hospital das Clínicas - Departamento de Cirurgia, $9^{\circ}$ Andar

Av. Bandeirantes, 3900 - Ribeirão Preto, CEP: 14048-900 